\title{
Classical conditioning of attitudes as a function of source consensus'
}

ROBERT FRANK WEISS, JOYCE JETTINGHOFF WEISS, \& LEO M. CHALUPA QUEENS COLLEGE OF THE CITY UNIVERSITY OF NEW YORK

Employing the general approach which Neal Miller (1959) has called "extension of liberalized $S-R$ theory," an analogy was drawn between source consensus in attitude formation and UCS-strength in classical conditioning. 350 Ss were exposed to persuasive arguments advocated by a group source with five levels of consensus $(0,25,50,75$, and $100 \%$ of "the experts"). As in classical conditioning, response probability was a negatively accelerated increasing function of "UCS-strength" (consensus) ( $p<.01)$.

Recent studies (e.g., Weiss, 1967; Weiss et al, 1963) have tended to support the application of instrumental conditioning and selective learning models to attitude formation (Weiss, 1962). The present paper explores the possibilities of a classical conditioning model (Weiss, 1962) in which the opinion to be learned is the conclusion of a syllogism. Ss are exposed to a persuasive communication which includes the premises of the syllogism, but not the conclusion (McGuire, 1960 ) and $S$ is left to draw the implied conclusion for himself (Hovland \& Mandell, 1952). Figure 1 shows corresponding paradigms for classical conditioning and attitude learning. Research based on this classical conditioning model requires persuasive communications which incorporate two elements: (1) the Opinion-Eliciting Argument (syllogism premises); and (2) the Cue Statement, two (neutral) words which immediately precede the opinion-eliciting argument and will later constitute part of the test used to measure attitude acquisition. The cue statement precedes the opionion-eliciting argument, so that an S listening to (or reading) the communication will first hear the cue statement, followed by the opinion-eliciting argument (syllogism premises), and then draw the conclusion implied by the argument. This sequence of events may be regarded as analogous to the sequence: CS, UCS, UR. The cue statement is the CS, and the opinion-eliciting argument is the UCS which elicits the implied opinion-the UR. Through repetition of the sequence the implied opinion (UR) becomes conditioned to the cue statement (CS) and thus becomes a conditioned opinion (CR) as shown in Fig. 1. Following the logic of this paradigm further, the power of the opinion-eliciting argument to convincingly imply an opinion is analogous to the strength of the UCS. The argument is attributed to a group source (experts), which is varied only in the degree of consensus among these experts (e.g., 25\% vs $100 \%$ of the experts). The degree of consensus among the sources of the argument affects the power of the argument to convincingly imply the opinion, and, hence, also corresponds to UCS strength. Research in classical conditioning (Ross \& Spence, 1960; Spence \& Platt, 1966) indicates that conditioned response strength (as measured by response probability) is a negatively accelerated increasing function of the strength of the UCS. Theoretically, then, attitude strength (as measured by response probability) should be a negatively accelerated increasing function of source consensus.

\section{Method and Procedure}

There were five levels of consensus: $0,25,50$, 75 , and $100 \%$ of "the experts," with $70 \mathrm{Ss}$ in each consensus condition $(\mathrm{N}=350)$. In each condition half the Ss were exposed to persuasive communication directed at opinion $A$, and half to persuasive communication directed at opinion B. A No Persuasion control condition was also included in the design. The Ss initially had "no opinion"' on both Opinion A and Opinion $B$ as measured by a questionnaire 2-8 weeks before the experiment. Persuasive communication was directed toward only one opinion for each $S$, but attitude toward both items was measured after persuasion. Thus, Ss who received a communication directed at Opinion $A$ served as controls for those who received a communication directed at Opinion B, and vice versa. (Order of testing of Opinion $A$ and Opinion B was counterbalanced.)

Opinion A was "Smetna's music is romantic in style." Opinion B was "The Lapps are descended from the ancient Mongols." The syllogism premises were embedded in longer passages which were ostensibly concerned with (A) nationalism and (B) linguistics. The consensus statements were attached to the second of the two premises presented in the communication, and were stated as proportions; e.g., "Three-fourths of the experts on Nineteenth century music are of the opinion that the music of nationalistic composers is romantic in style." (The first premise in this communication was: Smetna was a nationalistic composer.) Under the impression that he was participating in a study of "speech patterns and decision making" each $\mathrm{S}$ twice read the communication (and two other passages) into a tape recorder.
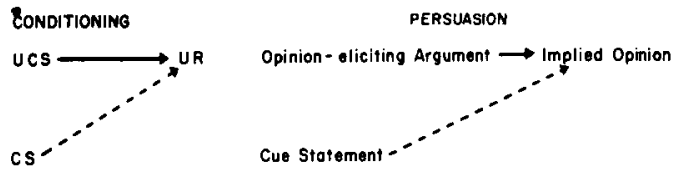

Fig. 1. Corresponding paradigms for classical conditioning and attitude learning. 


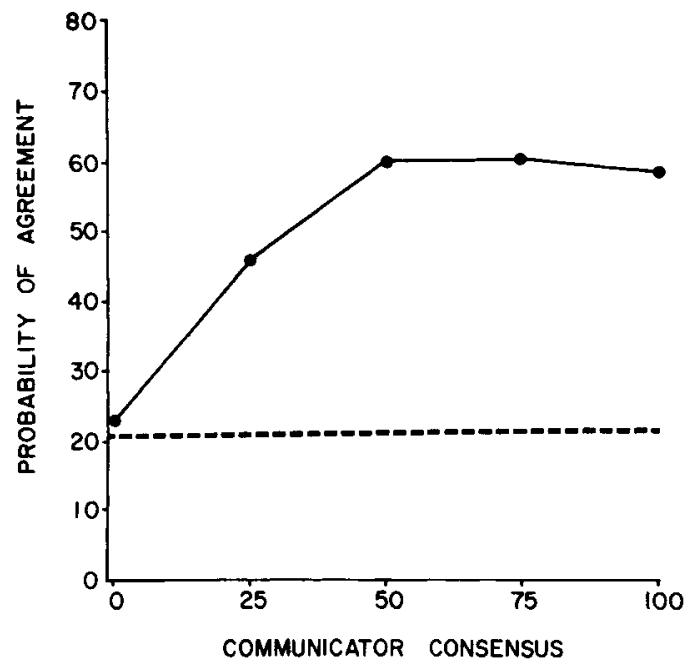

Fig. 2. Probability of agreement as a function of source consensus. (The dashed line represents performance in the no-persuasion control condition.)

In order to measure probability of agreement each $S$ was exposed to a card (preceded by 15 buffers) with the opinion (syllogism conclusion) at the bottom and the cue statement at the top. Ss were instructed to first look at the cue statement and then the opinion. They then estimated, aloud, the chances that the opinion was true on a scale from 0 to $100 \%$. Estimates of $51 \%$ and above were counted as indicating agreement, and the proportion of Ss in each group giving estimates of $51 \%$ and above was probability of agreement, the dependent variable.

\section{Results}

Figure 2 shows probability of agreement as a function of communicator consensus. The dashed line shows performance in the No Persuasion control condition. Even at the lowest level of consensus, opinions exposed to persuasive communication did not fall below the No Persuasion baseline. As predicted by the theory, probability of agreement was a negatively accelerated increasing function of communicator consensus. The consensus effect was statistically significant $\left(X^{2}=14.54, \mathrm{df}=4, \mathrm{p}<.01\right)$.

\section{Discussion}

The research reported here derives from a theory of attitude learning (Weiss, 1962) which is based on systematic analogies with learning research and Hull-Miller-Spence learning theory. The theory was formulated with sufficient detail to permit derivations which are not always intuitively obvious, such as interaction effects and curve shapes. Thus the theory predicted the consensus function found in this "classical conditioning" experiment. In a second study of the classical conditioning of attitudes, ${ }^{2}$ the following results also supported the theory: Attitude strength was an increasing function of argument strength (UCS strength) and number of persuasion trials, with these two variables combining multiplicatively to determine attitude strength. Results supporting the theory have also been found in "instrumental attitude learning." such as persuasion analogues of the delay of reinforcement gradient (Weiss, 1967) and the additive drive-reward interaction (Weiss et al, 1963). The source consensus variable may also find application to social psychological problems which do not derive from the present theory, such as group pressure studies on the Asch pattern, research on the effects of the views of "significant others" upon the selfconcept, and the quantitative variation of source credibility (Weiss et al, 1964).

\section{References}

HOVLAND, C. I., \& MANDELL, W. An experimental comparison of conclusion-drawing by the communicator and by the audience. J. abnorm, soc. Psychol, 1952, 47, 581-588.

McGUIRE, W. J. A syllogistic analysis of cognitive relationships. In M. J. Rosenberg, C. I. Hovland, W. J. McGuire, R. P. Abelson, \& J. W. Brehm. Attitude organization and change. New Haven: Yale Univer. Press, 1960. Pp. 65-111.

MILLER, N. E. Extensions of liberalized S-R theory. In S. Koch (Ed.), Psychology: a study of a science. Study 1, Vol. 2, New York: McGraw-Hill, 1959. Pp. 196-292.

ROSS, L. E., \& SPENCE, K. W. Eyelid conditioning performance under partial reinforcement as a function of UCS intensity. $J$. exp. Psychol, 1960, 59, 379-382.

SPENCE, K. W., \& PLATT. J. R. UCS intensity and performance in eyelid conditioning. Psychol Bull. 1966, 65, 1-10.

WEISS, R. F. Persuasion and the acquisition of attitudes: models from conditioning and selective learning. Psychol Rep., 1962, 11, 709-732.

WEISS, R. F. A delay of argument gradient in the instrumental conditioning of attitudes. Psychon. Sci, 1967, 8, 457-458.

WEISS, R. F., BUCHANAN, W., \& PASAMANICK, B. Social consensus in persuasive communication. Psychol Rep., 1964, 14, 95-98.

WEISS, R. F., RAWSON, H. E., \& PASAMANICK, B. Argument strength, delay of argument, and anxiety in the "conditioning" and "selective learning" of attitudes. J. abnorm. soc. Psychol, 1963, 67, 157-165.

Notes

1. Research supported by Grant MH-12402, National Institute of Mental Health.

2. Weiss, R. F., Chalupa, L. M., Gorman, B. S., \& Goodman, N. H. Classical conditioning of attitudes as a function of number of persuasion trials and strength of opinion-eliciting arguments. Unpublished study. 